\title{
Another role for nitric oxide in blood flow control?
}

\author{
Annemiek J. M. Cornelissen
}

Received: 4 March 2011/Accepted: 10 March 2011/Published online: 30 March 2011

(C) International Federation for Medical and Biological Engineering 2011

\begin{abstract}
In the current issue, Chen and co-authors present a mathematical model to simulate shear stressdependent nitric oxide (NO) transport in a small reconstructed microvascular network. Here their results are discussed in the context of NO-dependent blood flow control. Furthermore, other NO-dependent blood flow control mechanisms are briefly reviewed.
\end{abstract}

Keywords Blood flow control · Nitric oxide ·

Mathematical modeling · Microcirculation

My heart trouble will keep me here in Paris for another few days at least, until my doctors are in complete agreement about my immediate treatment. Isn't it the irony of fate that I have been prescribed N/G 1, to be taken internally! They call it Trinitrin, so as not to scare the chemist and the public.

These words wrote Alfred Nobel (1833-1896) in a letter to his friend Ragnar Sohlman on October 1896 just a couple of weeks before his death [18].

In his younger years [15] (1850), Alfred Nobel worked for a year in Theophile-Jules Pelouze's laboratory in Paris where he got acquainted with the discovery of a highly explosive compound, nitroglycerine (N/G 1) by Ascanio Sobrero 4 years earlier in the same laboratory. Alfred Nobel recognized the potential of this newly discovered chemical if he only could achieve a controlled detonation.

A. J. M. Cornelissen ( $\square)$

Laboratoire Matière et Systèmes Complexes (MSC), UMR 7057

CNRS \& Université Paris Diderot, Bât. Condorcet, 10, rue Alice Domon et Léonie Duquet, 75205 Paris cedex 13, France

e-mail: annemiek.cornelissen@univ-paris-diderot.fr
His experimentations together with his father back in Stockholm lead in 1863 to the Nobel patent detonator and eventually to a whole new class of explosives, dynamite. It is well known that after his death, according to his last will, his enormous fortune was used to institute the Nobel Prizes.

At the time, Alfred Nobel suffered from angina pectoris nitroglycerine was just about to be established as a treatment for the relief of angina pain [15]. But, Alfred Nobel declined the physicians' recommendation to take nitroglycerine as a medicine. It is well possible that he has been alarmed by the observations of Sobrera who was also the first to taste nitroglycerine and wrote: “...great precaution should be used, for a very minute quantity put upon the tongue produces a violent headache for several hours."

Despite Alfred Nobel's reservations, nitroglycerin became a reputable medicine for the treatment of angina pectoris. It took, however, about 100 years before the physiological mechanism of nitroglycerine was understood [21]. In 1977, Ferid Murad was analyzing how nitroglycerin works. He found that nitrates release nitric oxide (NO), which relaxes smooth muscle cells, resulting in vasodilation. The widened blood vessels allow more blood to pass. The improved circulation provides more oxygen for the heart muscle and the angina pain is thereby reduced.

In addition [21], Robert Furchgott concluded in 1980 that endothelial cells produce an unknown signal molecule that relaxes vascular smooth muscle cells. He called the signaling molecule endothelium-derived relaxing factor (EDRF). It was Louis Ignarro who in 1986 identified EDRF to be NO. In 1998, about 100 years after Alfred Nobel was suffering from severe Angina pains, Furchgott, Ignarro, and Murad were awarded the Nobel Prize in Physiology or Medicine for their discoveries concerning "the nitric oxide as a signaling molecule in the cardiovascular system." 


\section{Nitric oxide and remote blood flow control}

To my knowledge, Schretzenmayr [19] in 1933 was the first to speculate on remote vascular control of larger upstream vessels by production of local metabolic substances. He wondered how metabolic induced vascular dilation could be efficient when it is only limited to the perfusion areas that are in the direct vicinity and under direct influence of metabolic products. He argued that the resistance decrease due to local metabolic vasodilation could be much more efficient if also the larger upstream arteries would contribute. He indeed shows that after electrically triggering the femur muscle in the cat the femoral artery dilates. However, he did not unravel the signal that was responsible for this remote response.

Subsequently, it was shown that increased shear stress in the larger upstream arteries induced these remote responses, which was mediated by production of NO by the endothelial cells [11-13]. It should be noted that NO appears not to be the only molecule responsible for this endothelium-dependent vasodilation, also endotheliumderived prostaglandins [3] are involved as well as several endothelium-derived hyperpolarization factors [4]. Locally initiated hyperpolarizations by metabolic stimuli can be transmitted up- and downstream via gap junctions between smooth muscle and endothelial cells [10, 20].

Besides shear stress-induced NO-dependent vasodilation this conducted vasodilation contributes also to remote blood flow control.

In all, three main mechanisms for local control of vascular diameters have now been proposed: a pressuredependent basal myogenic response, a shear stressinduced vasodilation, and metabolic control. Mathematical modeling has added in understanding how these mechanisms contribute to flow control $[2,5,6,8,9,14]$. In these studies, diameter control is modeled for individual vessels, and the vascular tree is simplified in a number of representative compartments in series in which blood vessel segments of a certain diameter are arranged parallel. This modeling approach allows to analyze the individual diameter control mechanisms and to compare their activity at different levels in the vascular tree. In healthy basal metabolic situations, NO production coordinates the behavior of resistance vessels by lowering the level of basal myogenenic smooth muscle tone $[2,5,6,8$, $9,11]$. The modeling efforts $[2,4,5,10,20]$ show that this reduction in smooth muscle tone allows a wider spectrum to control vascular diameters and flow as when only myogenic and metabolic mechanisms would be involved. In the coronary circulation, the shear-dependent mechanism seems to most actively contribute to metabolic flow control in the larger arterioles (of about $100 \mu \mathrm{m})[8,14]$.

\section{Nitric oxide and local blood flow control}

In this current issue, Chen et al. [7] proposes another flow control mechanism that contributes to local redistribution of blood flow by diameter control of the precapillary arterioles $(<30 \mu \mathrm{m})$. They developed a mathematical model to investigate NO diffusion in tissue (notably to the vascular smooth muscle cells) perfused by a small reconstructed microvascular network of the hamster cheek pouch retractor muscle. They balance NO production by endothelial cells with the NO flux to the smooth muscle cells and NO consumption by red blood cells. They highlight the importance of the so-called red blood cell-plasma phase separation effect at vascular bifurcations [16, 17]. This effect, significant for arterioles smaller than $30 \mu \mathrm{m}$ [17], implies that the volume fraction of red blood cells that enters in the daughter branches is not proportional to the flow in these vessels. More precisely, at a bifurcation, the larger daughter branch receives proportionally a higher number of red blood cells than the smaller daughter branch. The results of Chen et al. suggest that this mechanism can have quite an effect on coronary blood flow control. They show when in a smaller daughter branch the flow decreases, NO production is decreased but so is the consumption rate in the blood owing to the decreased red blood cell hematocrit. The net effect is to lower NO levels in the vascular smooth muscle cell leading to a more constricted vessel, but not as much as without phase separation. Therefore, this mechanism rescues locally the tissue that is perfused by the side branch.

As a consequence of the phase separation effect and NO consumption by the red blood cells, NO-induced smooth muscle relaxation is able to equalize distribution of smooth muscle tone and flow even more than initially proposed [11]. It would be interesting to see how this mechanism contributes to flow control taking into account the other flow control mechanisms (remote control by shear stress induced diameter adaption, the myogenic response and other local metabolic responses). However, asymmetrical branching should be taken into account and instead of the empirical law for phase separation, derived from observations in the microvascular bed of the rat mesentery, a more generic physical law would be desirable to describe this effect for a larger range of flows.

Further interesting insights with respect to blood flow control are to be expected when besides NO transport, oxygen transport is modeled. Chen et al. models NO transport under normoxic conditions. There is recent intriguing evidence that under hypoxic conditions deoxygenating of hemoglobin stimulates NO release from the red blood cells resulting in vasodilation [1]. Further experimental and model studies are necessary to understand the coupling of oxygen sensing and NO release by hemoglobin 
and its functional role for flow control. The work of Chen and co-authors provides a solid base to model and understand these NO-dependent mechanisms.

\section{References}

1. Allen BW, Stamler JS, Piantadosi CA (2009) Hemoglobin, nitric oxide and molecular mechanisms of hypoxic vasodilation. Trends Mol Med 15(10):452-460

2. Arciero JC, Carlson BE, Secomb TW (2008) Theoretical model of metabolic blood flow regulation: roles of ATP release by red blood cells and conducted responses. Am J Physiol Heart Circ Physiol 295(4):1562-1571

3. Busse R, Forstermann U, Matsuda H, Pohl U (1984) The role of prostaglandins in the endothelium-mediated vasodilatory response to hypoxia. Pflugers Arch 401(1):77-83

4. Busse R, Edwards G, Feletou M, Fleming I, Vanhoutte P, Weston A (2002) EDHF: bringing the concepts together. Trends Pharmacol Sci 23(8):374

5. Carlson BE, Secomb TW (2005) A theoretical model for the myogenic response based on the length-tension characteristics of vascular smooth muscle. Microcirculation 12(4):327-338

6. Carlson BE, Arciero JC, Secomb TW (2008) Theoretical model of blood flow autoregulation: roles of myogenic, shear-dependent, and metabolic responses. Am J Physiol Heart Circ Physiol 295(4):1572-1579

7. Chen X, Buerk DG, Barbee KA, Jaron D (2011) 3-D network model of NO transport in tissue (Current issue)

8. Cornelissen AJM, Dankelman J, VanBavel E, Stassen HG, Spaan JAE (2000) Myogenic reactivity and resistance distribution in the coronary arterial tree: a model study. Am J Physiol Heart Circ Physiol 278(5):H1490-H1499

9. Cornelissen AJM, Dankelman J, VanBavel E, Spaan JAE (2002) Balance between myogenic, flow-dependent, and metabolic flow control in coronary arterial tree: a model study. Am J Physiol Heart Circ Physiol 282(6):2224-2237

10. de Wit C (2004) Connexins pave the way for vascular communication. News Physiol Sci 19:148-153

11. Griffith TM, Edwards DH, Davies RL, Harrison TJ, Evans KT (1987) EDRF coordinates the behaviour of vascular resistance vessels. Nature 329(6138):442-445

12. Holtz J, Forstermann U, Pohl U, Giesler M, Bassenge E (1984) Flow-dependent, endothelium-mediated dilation of epicardial coronary arteries in conscious dogs: effects of cyclooxygenase inhibition. J Cardiovasc Pharmacol 6(6):1161-1169

13. Kuo L, Davis MJ, Chilian WM (1995) Longitudinal gradients for endothelium-dependent and -independent vascular responses in the coronary microcirculation. Circulation 92(3):518-525

14. Liao JC, Kuo L (1997) Interaction between adenosine and flowinduced dilation in coronary microvascular network. Am J Physiol 272((4 Pt 2)):1571-1581

15. Marsh N, Marsh A (2000) A short history of nitroglycerine and nitric oxide in pharmacology and physiology. Clin Exp Pharmacol Physiol 27(4):313-319

16. Pries AR, Secomb TW (2005) Microvascular blood viscosity in vivo and the endothelial surface layer. Am J Physiol Heart Circ Physiol 289(6):2657-2664

17. Pries AR, Ley K, Claassen M, Gaehtgens P (1989) Red cell distribution at microvascular bifurcations. Microvasc Res 38(1): $81-101$

18. Ringertz N (2001) Alfred Nobel's health and his interest in medicine. http://nobelprizeorg/alfred_nobel/biographical/articles/ ringertz/. January 30, 2001

19. Schretzenmayr A (1933) Über kreislaufregulatorische Vorgänge an den großen Arterien bei der Muskelarbeit. Pflügers Archiv Eur J Physiol 232(1):743-748

20. Segal SS, Duling BR (1986) Flow control among microvessels coordinated by intercellular conduction. Science 234(4778):868-870

21. SoRelle R (1998) Nobel Prize awarded to scientists for nitric oxide discoveries. Circulation 98(22):2365-2366 